\title{
Tecnura
}

http://revistas.udistrital.edu.co/ojs/index.php/Tecnura/issue/view/640

DOI: http://dx.doi.org/10.14483/udistrital.jour.tecnura.2014.SE1.a21

Divulgación

\section{Comunidad científica y tecnológica, ha llegado la oficina que te acompañará en la transferencia de tu conocimiento}

\section{Scientific and technological community, The office that will accompany you in knowledge transfer has come}

\author{
Tatiana Monsalve Duarte*
}

Citation / Para citar este artículo: OTRI-Bogotá. (2014). Comunidad científica y tecnológica, ha llegado la oficina que te acompañará en la transferencia de tu conocimiento. Revista Tecnura, Edición especial, 273-276.

Fecha de recepción: 4 de febrero de 2014 / Fecha de aceptación: 11 de julio de 2014

La educación científica y la investigación aplicada se han venido constituyendo como ejes de prioridad en la generación actual, tanto en el mundo empresarial como en el social. Cada día crece la necesidad de fortalecer las bases de la creatividad para la producción de tecnología (tangible e intangible), apoyar al sistema con la integración de conocimiento aplicado y de esta manera hacer posible su materialización, por medio de redes de colaboración compuestas por especialistas conectados por un mismo fin: transferencia de conocimiento y tecnologías.

El trabajo que trae consigo un proceso de transferencia de conocimiento y tecnologías involucra la interacción de áreas especializadas como mercadeo para comercialización, ingeniería para vigilancia tecnológica, economía para valoración tecnológica y jurídica para propiedad intelectual, donde investigadores e inventores reciben la asesoría necesaria para preparar sus resultados de investigación e innovaciones como productos terminados para la sociedad.
Un gran objetivo es construir confianza en las ciencias que se encuentran detrás de cada concepto desarrollado bajo el resultado de una investigación. Los ciclos del aprendizaje contribuyen a la apropiación de conocimientos nuevos y con autonomía de desplazamiento, influyendo positivamente en el comportamiento individual y con la suma de ello, el de una sociedad; generando la cadena consecuente entre el pensar y el actuar. Lo que hace que la cultura en donde se genera este nuevo conocimiento, demande la expansión de la tecnología para lograr el aumento de su capacidad productiva y social.

Por ejemplo, los cambios de paradigmas de la generación actual con respecto a las anteriores han hecho que las fronteras entre países sean cada vez menos difusas, y con ello el deseo de conocer una segunda lengua, gracias a la necesidad de adaptación a culturas diferentes. La tecnología en la actualidad soporta la creación de dispositivos, programas y diversas aplicaciones con las que una persona sin necesidad de pagar por su uso pueda aprender diferentes lenguas de

\footnotetext{
* Especialista de marketing y relaciones de la Oficina de Transferencia de Resultados de Investigación de Bogotá, OTRI-Bogotá. Máster en Gestión y Dirección de Empresas Internacionales.
} 
manera autodidacta; atravesar fronteras y comunicarse de manera visual o escrita, por medio de envío de información y datos al utilizar diversos mecanismos, que en tiempos pasados no se hubiesen imaginado.

Las personas tienen la capacidad de inventar, crear y construir a través de la necesidad o simplemente del deseo por algo, lo que hace que el cerebro trabaje constantemente, y de esa manera trazar el objetivo en que esas percepciones diarias y conocimiento se traduzcan en beneficios para una comunidad o el cubrimiento de una necesidad empresarial.

El gobierno de Estados Unidos como uno de los principales emprendedores de transferencia formal de tecnología, reconoció en su momento la necesidad de crear una oficina que se especializara y se dedicara solamente a identificar, desarrollar, promover y negociar sobre el conocimiento generado por los investigadores e inventores, con el fin de entregarlo a la comunidad para obtener resultados aplicados a la producción y comercialización de los productos creados e innovados.

Bajo este modelo propuesto es como trasciende las fronteras el concepto de Oficina de Transferencia de Resultados de Investigación (OTRI), la cual se especializa en generar procesos eficientes para que la tecnología (tangible o intangible) y/o innovaciones se encuentren en las condiciones pertinentes, y así los usuarios finales disfruten de los beneficios generados a través de esa transferencia.

En Colombia ya se conocía la existencia de oficinas de transferencia en algunas universidades, interesadas precisamente en impulsar los resultados de las investigaciones gestadas en su interior; sin embargo, no era un concepto que se manejara de manera frecuente ni muy conocido por las personas que se encontraban involucradas en investigación o en comercialización de conocimiento. Es así como Colciencias promueve a través de su convocatoria 621 de 2013, la creación y fortalecimiento de Oficinas de Transferencia de Resultados de Investigación (OTRI) en diferentes regiones del país, las cuales debían ser formuladas mediante alianzas entre universidades, centros de investigación, centros de desarrollo económico y empresas de diferentes sectores; donde se encontrarían involucrados especialistas con experiencia en la gestión de protección intelectual, vigilancia tecnológica, valoración de intangibles y tecnologías y, por último, la comercialización, promoción y conexión con el mundo inversionista y empresarial, con el objetivo de dar vida a cada resultado de investigación, cada tecnología y/o invento, generando impacto positivo en la población objetivo.

La iniciativa de crear la OTRI-Bogotá nace de la Universidad Distrital Francisco José de Caldas (desde su Centro de Investigaciones y DesarroIlo Científico), en co-ejecución con la Secretaría Distrital de Desarrollo Económico (desde su Subdirección de Ciencia, Tecnología e Innovación), como esfuerzo conjunto para así no dejar pasar la oportunidad que Colciencias exponía a través de su convocatoria y de esta manera aprovechar las condiciones favorables de investigación y cobertura con las que la Universidad Distrital y la Secretaría cuentan para la creación e implementación de la Oficina. La OTRI-Bogotá tiene como objetivos fundamentales facilitar la transferencia de resultados de investigación, innovación y tecnologías, gestadas por investigadores e inventores de Bogotá - Región, hacia el sector productivo, permitiendo así visibilizar, promover y desarrollar la materialización de un producto vendible y rentable, social y económicamente.

El acompañamiento y asesoría son posibles mediante la prestación de los siguientes servicios:

\section{Marketing y Transferencia Tecnológica}

Comprensión y conocimiento de manera precisa sobre las ventajas competitivas de la tecnología.

- Validación técnico-comercial de la tecnología.

- Análisis de la competencia en sus diferentes frentes. 
- Comunicación y divulgación de los beneficios de la tecnología.

- Creación de las conexiones necesarias para realizar la transferencia.

\section{Vigilancia y Diagnóstico de la Madurez de la Tecnología}

Investigación organizada, selectiva y permanente de información sobre ciencia y tecnología, para convertirla en la base de toma de decisiones.

- Informes de vigilancia tecnológica e inteligencia competitiva (basados en la Norma UNE 1666006:2006).

- Informes de competencia, estado del arte y estado de la técnica.

- Búsqueda de antecedentes nacionales e internacionales y sus patentes equivalentes.

- Evaluación del estado de desarrollo de las tecnologías.

- Informes de prospectiva y tendencias.

- Alertas de invenciones e innovación.

\section{Valoración Tecnológica}

Conocimiento de la tecnología y su valoración (tangible e intangible), no a partir de lo que cuesta sino de lo que es posible producir a través de ella.

- Evaluación de la relación entre la propiedad intelectual, la fuerza de su comercialización y la tecnología.

- Conocimiento contable (entendimiento y cálculo de costos de producción).

- Valor Presente Neto (gestión y evaluación de proyectos).

- Comprensión del mercado (comparaciones con tecnologías existentes y similares).

- Conocimiento de las opciones reales (basado en las opciones financieras).

- Modelación de negocios (acorde con el tipo de protección).

\section{Propiedad Intelectual y Protección}

Asesoría especializada para el aseguramiento de la tecnología, de acuerdo con su innovación y con respecto a la competencia.

- Diagnóstico del tipo de protección necesaria y pertinente.

- Creación del modelo de negociación y así dar solidez a la tecnología.

- Protección de las invenciones, creaciones y marcas para prevenir las utilizaciones no autorizadas en el comercio de los productos.

El proceso para la creación e implementación de la OTRI-Bogotá ha sido acompañado por Colciencias y su agenda de entrenamiento, dirigida al fortalecimiento de los especialistas que componen cada una de las OTRI a nivel nacional (Atlántico, Santander, Antioquia y Cundinamarca), soportada por la consultoría de los señores Glenn E. Robinson y Norman Kaderlan, del IC2 de la Universidad de Texas en Austin.

Gracias a una preparación previa mediante el programa virtual "Innovation Readiness de IC2 Colciencias", donde los especialistas recibieron la formación necesaria para profundizar sus conocimientos en cada una de las áreas, basado en la metodología Quicklook y creación de Oficinas de Transferencia de Resultados de Transferencia, aplicando un enfoque neto sobre transferencia de tecnología; con el objetivo de crear redes de trabajo e intercambio de saberes entre OTRIs a nivel nacional, la primera semana de julio de 2014 se realizó el primer encuentro de OTRIs a nivel nacional, para consolidar los conocimientos adquiridos del programa de manera presencial y práctica, logrando el objetivo de enlace y comunicación entre OTRIs.

Adicionalmente la OTRI-Bogotá, se encuentra desarrollando asesoría y acompañamiento a diferentes tecnologías, que ya se han acercado con sus prototipos y productos terminados, para lograr facilitar la transferencia y entrega al mundo de los negocios. Entre los productos que se encuentran 
actualmente en el portafolio, se trabaja en el proceso de transferencia de dispositivos y aparatos eléctricos para el mejoramiento de la calidad de vida de personas que presentan algún tipo de discapacidad, innovaciones en artefactos que mejoran los procesos de producción de las empresas y con ello, el beneficio de generación de economía, entre otros que impactan a segmentos especializados y masivos de la comunidad.

Actualmente se encuentra realizando gestión para la firma de acuerdos de cooperación con empresas, universidades y Oficinas de Transferencia de Conocimiento en países como México, España, Bolivia y Argentina. El pasado 26 de Noviembre de 2014, se firmó la carta de intención de Querétaro en México, donde se expresa la voluntad de cooperación entre la transferencia de conocimiento entre los países de Costa Rica, Guatemala, Brasil, México, Argentina y en representación de Colombia, OTRI Bogotá.

Gracias al trabajo realizado en equipo y al gran apoyo del Coordinador de la OTRI-Bogotá el Ingeniero José Nelson Pérez Castillo, la operación de la Oficina se desarrolla y crece a buen ritmo.

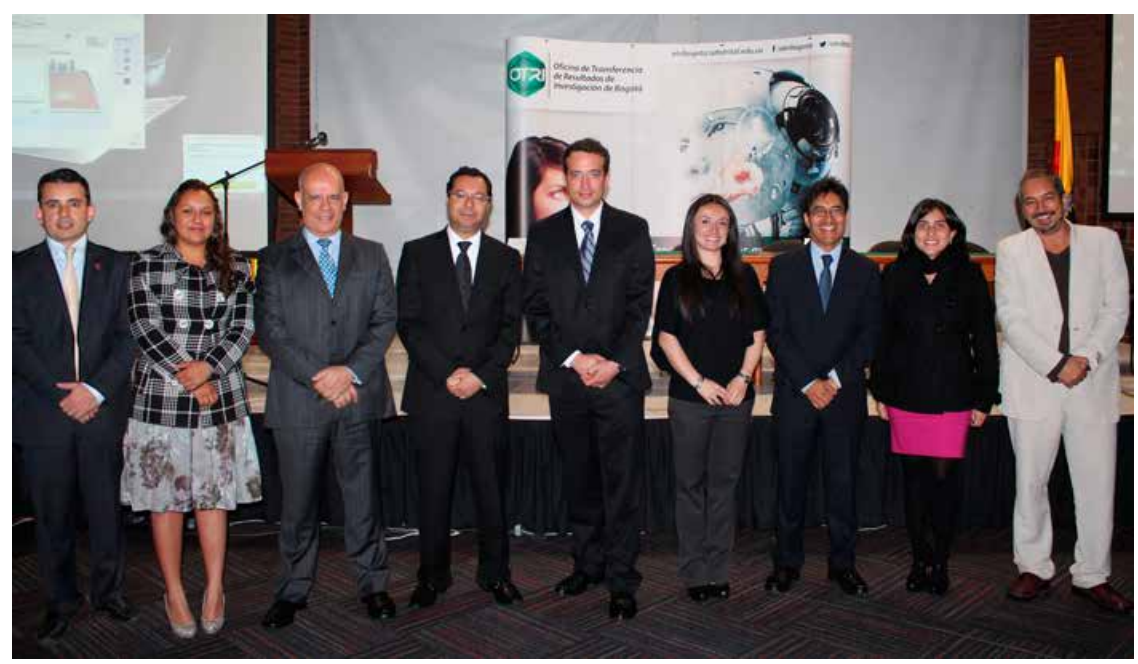

Lanzamiento de la OTRI-Bogotá, 11 de Diciembre de 2011, con las ponencias de expertos en transferencia tecnológica y empresarios de innovación.

Izquierda a Derecha:

Pedro Fabián Pérez (Vigilancia Tecnológica), Ivanna García (Infotec, México), Luiz Otavio
Pimentel (Universidad Santa Catarina, Brasil), Juan Carlos Serna (Propiedad Intelectual), Olivier Buigues (Obinova, España), Tatiana Monsalve (Marketing y Relaciones), Jairo Rodríguez (Valoración Tecnológica), Andrea Rojas (Directora de Proyectos OTRIs, Colciencias) y José Nelson Pérez CastiIlo (Coordinador OTRI-Bogotá)

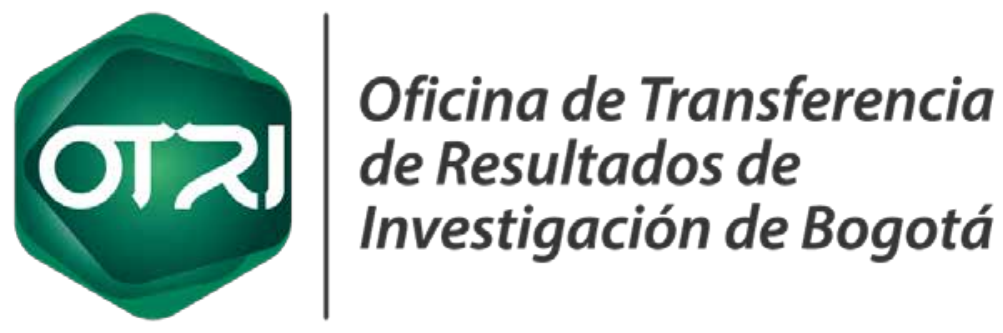

Tecnura • p-ISSN: 0123-921X • e-ISSN: 2248-7638 • Edición especial 2014 • pp. 273-276 